
Revue
de I'histoire
Revue de l'histoire des religions
des religions
$4 \mid 2005$
Lieux de culte, lieux saints dans le judaïsme, le christianisme et l'islam

\title{
Le rôle des tombes sacrées dans la conquête ottomane
}

The Part held by Sacred Graves in Ottoman Conquest

\section{Gilles Veinstein}

\section{(2) OpenEdition \\ Journals}

Édition électronique

URL : http://journals.openedition.org/rhr/4228

DOI : $10.4000 /$ rhr.4228

ISSN : 2105-2573

Éditeur

Armand Colin

Édition imprimée

Date de publication : 1 octobre 2005

Pagination : $509-528$

ISBN : 2200-92087-3

ISSN : 0035-1423

Référence électronique

Gilles Veinstein, « Le rôle des tombes sacrées dans la conquête ottomane », Revue de l'histoire des religions [En ligne], 4 | 2005, mis en ligne le 18 janvier 2010, consulté le 01 mai 2019. URL : http:// journals.openedition.org/rhr/4228; DOI : 10.4000/rhr.4228 


\section{GILLES VEINSTEIN}

Collège de France, Paris

École des Hautes Études en Sciences Sociales, Paris

\section{Le rôle des tombes sacrées dans la conquête ottomane}

L'article part d'une constatation : plusieurs récits plus ou moins historiques ou légendaires mentionnent parmi les événements accompagnant une nouvelle conquête ottomane, la découverte ou la réhabilitation de la tombe d'un saint personnage de l'islam, préalablement dissimulée ou négligée. Symétriquement, d'autres traditions évoquent l'inhumation d'un précurseur dont la tombe en terre infidèle est le gage d'une conquête musulmane future. Nous nous interrogeons sur les origines et la signification d'un phénomène aussi récurrent. Nous sommes conduit à une vision plus complexe que les seules provisions de la Cherî'a de ce qu'impliquaient pour les Ottomans la légitimité d'une conquête et l'islamisation d'un nouveau territoire.

\section{The Part held by Sacred Graves in Ottoman Conquest}

This article takes root in an observation: several narratives, more or less historical or legendary, mention among events surrounding a new Ottoman conquest, the discovery or the rehabilitation of the grave, hidden or neglected up to then, of a holy Islamic personality. Symmetrically, other traditions evoke the burying of a forerunner whose grave in infidel grounds represents a token for a future Muslim conquest. Considering the origins and the signification of such a recurrent phenomenon, we are led to believe that the legitimacy of a conquest and the islamization of a new territory are more complex than what is implied in the Sherî'a. 
La réflexion à laquelle je vais me livrer sur le rôle des tombeaux dans la conquête ottomane est née de l'observation d'un aspect singulier qui se répète dans plusieurs phases de cette conquête. Je veux parler du fait qu'au moment où l'un des sultans ottomans conquiert une nouvelle province, on redécouvre pour lui ou du moins on remet à l'honneur à cette occasion le tombeau d'un saint personnage. Ce dernier avait été inhumé là, plus ou moins longtemps avant cette conquête et sa sépulture avait été par la suite occultée ou négligée. Je vais, pour commencer, m'attacher à quatre illustrations bien connues de ce phénomène.

\section{DES CONQUÊTES LIBÉRATRICES DE TOMBES}

Mon premier cas est relié à la conquête de Constantinople par Mehmed II en 1453. Une tradition élaborée postérieurement aux faits et dont Joseph von Hammer se fait l'écho dans sa célèbre Histoire de l'Empire ottoman, veut que ce soit pendant le siège, donc avant même la conquête proprement dite, que les Turcs aient retrouvé, à l'extérieur des murailles de la ville, la sépulture d'Abû Ayyûb (Eyüp en turc), compagnon et porte-étendard du Prophète ${ }^{1}$. Ce dernier aurait péri là, lors d'une des tentatives des premiers conquérants arabes contre la capitale de l'Empire byzantin - tentative située, toujours selon la tradition, en 52 de l'Hégire (672). En fait, parmi les raids arabes contre Constantinople historiquement établis, le plus proche, chronologiquement, est celui de Yazîd b. Mu'âwiya, qu'il faut placer entre 47 et 49 de l'Hégire, soit vers 667-669, avec cette réserve pourtant que le raid en question semble s'être arrêté, en réalité, sur la rive asiatique du Bosphore ${ }^{2}$.

Des investigations historiques plus rigoureuses, menées sur cet épisode, apportent deux enseignements principaux : premièrement, c'est en fait après la conquête de Constantinople, selon toute

1. Joseph de Hammer, Histoire de l'Empire ottoman depuis son origine jusqu'à nos jours, trad. Jean-Jacques Hellert, II, Paris, 1835, p. 394-395.

2. Marius Canard, "Les expéditions des Arabes contre Constantinople dans l'histoire et dans la légende », Journal Asiatique, CVIII, 1926, p. 68-77. 
probabilité vers 1456-1457, qu'eut lieu «l'invention » du tombeau d'Eyüp auquel le conquérant ottoman consacra un mausolée, accompagné d'ailleurs d'une grande mosquée et d'un tekke de derviches, ce complexe étant appelé à devenir le célèbre lieu de pèlerinage pour les musulmans stambouliotes et pour l'ensemble des musulmans, qu'on connaît encore aujourd'hui. D'autre part, il apparaît qu'il y eut en fait une controverse sur le véritable emplacement de la sépulture d'Abû Ayyûb, que Mehmed II avait fait rechercher par son maître spirituel, le cheikh Akshemseddîn. Si les uns optaient pour le lieu que retiendra finalement le sultan, situé au fond de la Corne d'Or et surplombant Istanbul, d'autres - et parmi eux, à ce que précisent certaines sources, les oulémas - considéraient que le véritable lieu de la mort et de l'inhumation du compagnon du Prophète, se trouvait à l'intérieur même de la ville. Plus précisément, ils le situaient dans un endroit que l'historien Paul Wittek, sur la base des descriptions fournies par les rares sources, identifiait avec l'enclave des Blachernes, au nord du palais byzantin du même nom, entre les murailles d'Héraclius et de Léon V. Cet emplacement désigné sous l'appellation de Pentapyrgion correspondrait à l'actuel quartier d'Ayvansaray (dont l'étymologie renverrait précisément à l'expression Ayyûb Ansârî). Pour apaiser les esprits, le successeur de Mehmed II, Bâyezîd II, aurait fait de ce second emplacement, écarté par le conquérant, un lieu saint secondaire, celui de l'inhumation du frère d'Abû Ayyûb et d'un grand nombre de ses compagnons, également voués au martyre. Il est d'ailleurs à relever que les deux endroits envisagés correspondaient l'un et l'autre à des lieux saints pré-islamiques : une ancienne fontaine sacrée dite de Saint Basile dans le cas d'Ayvansaray ; une ancienne chapelle chrétienne, probablement dédiée à Saint Côme et Saint Damien, dans celui du lieu finalement retenu, l'actuel sanctuaire d'Eyüp ${ }^{3}$.

3. Paul Wittek, « Ayvansaray. Un sanctuaire privé de son héros », Annuaire de philologie et d'histoire orientales et slaves, XI, Mélanges Henri Grégoire III, Bruxelles, 1951, p. 505-526 ; reprint in Paul Wittek, La formation de l'Empire ottoman, Variorum Reprints, 1982 ; A. I. Yurd et M. Kaçalın, Akşemseddin Hayati ve Eserleri, Istanbul, 1994 ; Stéphane Yerasimos, "La ville ottomane de 1453 à la fin du XVIII ${ }^{\mathrm{e}}$ siècle ", in Gilles Veinstein, Marie-France Auzépy, Alain Ducellier, Stéphane Yerasimos, Istanbul, Paris, 2002, p. 175-176. 
La conquête de la Syrie par Selîm $1^{\text {er }}$ en 1516, qui sera suivie l'année d'après par la conquête de l'Égypte, donne lieu à son tour à la construction d'un mausolée, assorti d'une mosquée. Il s'agit cette fois du tombeau d'Ibn 'Arabî dans le quartier de Salahiyya à Damas. L'emplacement de la tombe du grand mystique, mort à Damas, n'avait jamais cessé d'être connu. Mais le discrédit de ce penseur auprès des oulémas damascènes, sous l'influence du courant hanbalite et des critiques acerbes de cet autre penseur damascène que fut Ibn Taymiyya, explique que cette tombe avait été laissée à l'abandon et qu'elle était même, selon certains témoignages, recouverte d'immondices. C'est à son retour d'Égypte que Selîm avait entrepris, non sans paradoxe si l'on se place du point de vue des docteurs rigoristes de la place, d'honorer le "Sheikh al-Akbar», en édifiant sur ses restes un imposant complexe architectural ${ }^{4}$.

Le lien entre conquêtes et édifications de tombeaux, réapparaît sous le règne suivant, celui de Soliman le Magnifique : lorsque celui-ci s'empare de Bagdad en 1534, arrachant cette ville à la domination des Safavides chiites, il n'a rien de plus pressé que de faire rechercher le tombeau du grand juriste Abû Hanîfa qui a donné son nom à l'école juridique dont les Ottomans, comme les autres dynasties turques, se réclamaient, ainsi que les restes de l'éminent docteur que les Chiites avaient pillés et profanés. Nous apprenons que le grand imam étant apparu en songe au gardien du tombeau et lui ayant commandé de sauver ses restes de la profanation des hérétiques, ce dernier avait opportunément remplacé le corps du bienheureux par celui d'un infidèle, et l'avait déposé en lieu sûr. Ibrâhîm Pacha grand vizir de Soliman, ayant informé son maître de cette bienheureuse circonstance, le sultan avait chargé un savant, le müderris Tashkun, de se mettre en quête de la sainte relique. Celui-ci annonce bientôt qu'à l'endroit qu'on lui avait désigné, les ouvriers avaient rencontré, en remuant la terre, un mur d'où s'était exhalée une forte odeur de musc - preuve sans équivoque que les restes de l'imam étaient bien là et que le gardien du tombeau avait dit vrai.

4. Cf. Ryad Atlagh, "Paradoxes d'un mausolée», in Mohammad Ali Amir-Moezzi, éd., Lieux d'islam. Cultes et cultures de l'Afrique à Java, Paris, 1996, p. 132-153. 
Soliman et son vizir se rendent alors sur place. Ibrâhîm retire de ses propres mains la pierre qui dissimulait l'entrée du mausolée. Les deux hommes descendent sous la voûte de l'édifice et découvrent par eux-mêmes les restes d'Abû Hanîfa. Toute l'armée se rend en pèlerinage au tombeau de l'imam et Soliman ordonne la construction d'une coupole au-dessus de ces restes, le mausolée ainsi édifié devenant un lieu de pèlerinage pour les sunnites de Bagdad et du reste de l'Irak ${ }^{5}$.

Le quatrième cas que j'évoquerai enfin est celui du mausolée, accompagné d'un tekke de derviches, dédié à Umm Hizâm, épouse de 'Ubâda b. al-Sâmit, une parente du Prophète, sur les salines de Larnaca, à Chypre. Cette sainte femme passait pour être morte sur cette île, après être tombée du chameau ou bien du mulet sur lequel elle était montée, en prenant part à l'expédition contre l'île de Mu'âwiya, gouverneur de Damas, en 647, et pour avoir été enterrée sur place. Les troupes de Selîm II s'emparent de l'île en 1571, mettant fin à la longue domination chrétienne des Byzantins, des Francs de la dynastie des Lusignan, puis des Vénitiens. Plus ou moins longtemps après cet événement, à une date que je ne suis pas en mesure de préciser, ce complexe voit le jour, sous le nom de tekke de Hâla Sultân, et devient un lieu de pèlerinage pour les musulmans chypriotes ${ }^{6}$.

Bien entendu, les cas que je viens d'énumérer ne sont pas entièrement assimilables les uns aux autres. Chacun de ces épisodes se situe dans un contexte historique particulier et est susceptible d'interprétations spécifiques. En relevant la tombe d'Abû Ayyûb et en préférant l'emplacement extérieur aux murailles à celui qui se trouvait à l'intérieur de la ville, Mehmed II, peut-on aisément imaginer, mettait en évidence qu'il avait réussi là où les compagnons du Prophète et les premiers conquérants arabes avaient échoué; qu'il parachevait en quelque sorte leur œuvre et qu'il s'inscrivait ainsi dans la continuité de l'histoire la plus glorieuse et la plus sacrée.

Selîm $1^{\text {er }}$, quant à lui, devait faire face à l'illégitimité foncière de la guerre impitoyable, entachée de pillages et de massacres, qu'il

5. Hammer, op. cit., V, Paris, 1836, p. 222-223.

6. Cf. A. C. Gazioğlu, The Turks in Cyprus, Londres, 1990, p. 279. 
avait menée contre les Mameloukes, des musulmans sunnites comme lui et qui avaient les états de service les plus brillants pour la cause de l'islam dont ils avaient été le rempart, aussi bien contre les païens mongols que contre les croisés. Je formule ainsi l'hypothèse qu'en mettant à l'honneur la figure d'Ibn 'Arabî que l'Etat ottoman, dès ses origines, avait placé au premier rang des docteurs, il cherchait à contrebalancer la réprobation qu'il s'était attirée de la part des oulémas syriens et égyptiens, en se revendiquant d'une autorité religieuse d'une inspiration différente, mais au moins aussi vénérable et légitime ${ }^{7}$. Cette autorité, en effet, avait, par avance, justifié son action en prédisant la conquête ottomane des pays arabes et surtout de l'Égypte, dans un ouvrage de jafr qui annonçait, plusieurs siècles à l'avance, la fortune des Ottomans et le rôle, quasi messianique, de Selîm lui-même ${ }^{8}$. Il ne fait aucun doute que l'ouvrage en question, Al-shadjara al-nu'mâniyya fil-Dawla al-'uthmâniyya, était en réalité un faux. Précisément, peut-on supposer, son élaboration, du moins dans son noyau initial, fit partie de la même « entreprise idéologique » que l'érection du mausolée. De fait, on constate que la visite à ce mausolée compta désormais parmi les premiers devoirs des gouverneurs ottomans de Syrie nouvellement en poste; que la mosquée qui le jouxtait devint une sorte de mosquée officielle du régime ottoman et, enfin, que plusieurs des gouverneurs ottomans, morts en fonction à Damas, furent inhumés dans le cimetière voisin, à proximité des restes du grand cheikh ${ }^{9}$.

7. Ce qui se conçoit en tenant compte de la reconnaissance dont la doctrine d'Ibn 'Arabî a fait l'objet très tôt et durablement de la part de la dynastie ottomane; cf. Michel Chodkiewicz, «La réception de la doctrine d'Ibn 'Arabî dans le monde ottoman », in Ahmed Yakşar Ocak, éd., Turkish Sufism in Historical process, Ankara, à paraître. Tous mes remerciements vont à l'auteur qui m'a communiqué son manuscrit encore inédit.

8. Cf. Denis Gril, «L'énigme de la Şă̆ara al-nu'māniyya fî l-dawla al- 'utmāniyya attribuée à Ibn 'Arabī » in Benjamin Lellouch et Stéphane Yerasimos (éds.), Les traditions apocalyptiques au tournant de la chute de Constantinople, Paris, 1999, p. 133-151.

9. Cf. Henri Laoust, Les gouverneurs de Damas sous les Mamelouks et les premiers Ottomans (658-1156/1260-1744), traduction des Annales d'Ibn Ṭūlūn et d'Ibn Ğum'a, Damas, 1952. 
La réhabilitation du tombeau de Abû Hanîfa à Bagdad, symbolisait, de toute évidence, le rétablissement de l'orthodoxie sunnite dont ce juriste était la figure emblématique pour les Ottomans, dans l'ancienne capitale califale sur laquelle les Safavides avaient fait préalablement régner l'hérésie chiite.

Quant à la tombe de Umm Hizâm à Chypre, elle était une manière de rappeler que l'île qui avait été successivement placée sous plusieurs dominations chrétiennes, avait été néanmoins, dans des temps lointains (entre le $\mathrm{VII}^{\mathrm{e}}$ et le $\mathrm{x}^{\mathrm{e}}$ siècle), dont, manifestement, le souvenir ne s'était pas effacé dans la mémoire musulmane, une sorte de condominium entre le calife et le basileus byzantin ${ }^{10}$. Ce point était d'importance pour les Ottomans puisque s'en prendre à Chypre n'était pas accomplir n'importe quelle conquête : ils n'avaient pu entreprendre celle-ci qu'en violant le traité ('ahdnâme) qui les unissait aux Vénitiens et que les sultans successifs (en dernier lieu, Soliman le Magnifique, en 1540) avaient fait serment de respecter ${ }^{11}$.

Destinée à apporter une solution à ce problème juridique et moral, la fetvâ émise par l'illustre mufti d'Istanbul, Ebû Su'ûd efendi, était fondée sur ce passé islamique de Chypre, qui, en dernière analyse, conférait tous les droits au sultan. Le préambule de la question posée à Ebû Su'ûd, était formulé ainsi :

«Une terre appartenait antérieurement au dâr al-islâm. Après un certain temps les vils infidèles l'ont envahie. Ils ont mis en ruines les collèges et les mosquées et les ont vidés. Ils ont rempli les chaires et les galeries des marques de la mécréance et de l'erreur, avec l'intention de faire insulte à la religion de l'islam, en se livrant à toutes sortes de mauvaises actions. Sa Majesté le pâdichâh, refuge de la Religion a décidé, comme le requiert la protection de l'islam, d'enlever le pays en question des mains des Infidèles méprisables et de l'annexer au dâr al-islâm. »

À cette occasion, ce manquement au serment était justifié par le fait que des édifices musulmans avaient existé autrefois sur cette île

10. Encyclopédie de l'Islam 2, t. V, art. « Kubrus », p. 301-302.

11. Texte de la capitulation ('ahdnâme) de 1540 dans laquelle Soliman le Magnifique prête serment à deux reprises de respecter les possessions vénitiennes, in Mehmed Tayyib Gökbilgin, « Venedik Devlet Arşivindeki Vesikalar Külliyatında Kanunî Sultan Süleyman Devri Belgeleri », Belgeler, I, 2, juillet 1964, Ankara, 1965, p. 121-128. 
et que les mécréants qui l'avaient dominée ensuite les avaient profanés. L'honneur de l'islam devait donc, en l'occurrence, l'emporter sur toute autre considération. Le Prophète en avait donné lui-même l'exemple en rompant deux ans après le pacte qu'il avait conclu avec les Infidèles de La Mecque, dans la sixième année de l'Hégire, quand l'intérêt supérieur de l'islam l'avait exigé.

Dans sa réponse, le mufti affirmait qu'il n'y avait aucun obstacle à rompre avec Venise ce qui n'avait jamais été qu'une trêve, dès lors que l'islam en tirait bénéfice.

Ainsi la tombe de la tante du Prophète pouvait être considérée comme un exemple privilégié de ces vestiges musulmans qu'il fallait relever et préserver des profanations des Infidèles ${ }^{12}$.

Les cas de tombes redécouvertes et honorées à l'occasion d'une conquête ne s'arrêtent pas à l'énumération qui précède. D'autres exemples seraient certainement à citer et il conviendrait d'ailleurs d'en dresser une liste plus complète. Peu importe d'ailleurs que ces épisodes correspondent à des faits réels ou qu'il s'agisse de légendes forgées après coup. Dans les deux cas, les mêmes croyances sont à l'œuvre, qu'il s'agit pour nous de tenter de cerner. Le voyageur Evliyâ Çelebi, grand collecteur de mythes et introducteur incomparable aux mentalités ottomanes, rapporte ainsi un fait, s'inscrivant dans la même ligne que les précédents, qui n'a rien d'invraisemblable dans son principe, mais qui cependant, est ignorée des chroniqueurs contemporains, et pourrait bien n'être qu'une invention postérieure. Lors de sa « campagne de Moldavie » (Sefer-i Karabogdan) de 1484, Bâyezîd II aurait redécouvert à Babadagh la tombe de Sarı Saltuk, guide des Turcs établis en Dobroudja à l'époque des Seljoukides de Rûm. Le saint lui serait alors apparu et, dans une prophétie qui n'est pas sans rappeler celle attribuée à Ibn 'Arabî dans la shadjara, il lui aurait annoncé qu'il allait conquérir les deux cités moldaves de Kili (Chilia) et Akkerman (Cetatea Alba) — ce qu'il fit en effet

12. Sur la fetvâ d'Ebû Su'ûd, cf. M. Ertuğrul Düzdağ, Şeyhülislâm Ebussu'ûd Efendi fetvaları Işı̆ğında 16. Astr Türk Hayatı, Istanbul, 1983, n ${ }^{\circ}$ 478, p. 108-109 ; Colin Imber, Ebu's-Su'ud. The Islamic Legal Tradition, Edinburgh, 1997, p. 84-85. 
peu après —, mais également qu'il reviendrait à ses fils de posséder La Mecque et Médine ${ }^{13}$.

À côté des différences qui sont à relever entre les diverses situations que nous venons d'énumérer et des significations particulières qu'il convient d'accorder à chacune d'entre elles, le lien quasi automatique entre investissement d'un nouveau territoire et remise en valeur d'un tombeau renvoyant aussi bien à l'histoire qu'à la légende, est en tout état de cause le point commun à tous ces épisodes. À chaque fois le nouveau territoire dont s'empare le conquérant n'apparaît pas comme un territoire vierge, un désert du point de vue de la foi, pénétré uniquement de mécréance ou d'hérésie. Recélant au contraire en son fonds la dépouille d'un saint personnage, plus ou moins illustre, il portait déjà en lui le germe sacré du salut ou de la réhabilitation sous le règne de la vraie foi. Ce saint mort apparaît ainsi, rétrospectivement, comme le précurseur du conquérant, et celui-ci à son tour n'est pas une génération spontanée bâtissant ex nihilo : il lui revient de parachever l'œuvre que le premier n'avait fait qu' amorcer. Il n'est pas en position de novateur mais bien de continuateur.

\section{DES TOMBES PROMETTEUSES DE CONQUÊTES}

D'autres traditions liées à la conquête ottomane ou plus largement à la conquête turque, offrent l'intérêt de nous ramener en amont, dans cette phase antécédente postulée par les événements rapportés jusqu'ici. Elles en éclairent ainsi la signification.

Dans le Danishmendnâme, épopée rédigée au XIV siècle pour relater ses hauts faits, Gâzî Melik Danishmend, l'un des conquérants de l'Anatolie, fondateur d'une principauté turkmène au centre de la péninsule, voit le Prophète lui apparaître en songe, pour lui tenir ces propos :

«Maintenant, il te faut mourir [...] À ton réveil, tu devras quitter tes compagnons $[\ldots]$ Ils devront t'enterrer. Il faut que ton corps reste ici

13. Cité par Machiel Kiel, "Ottoman Urban Development and the cult of a heterodox Sufi Saint: Sarı Saltuk Dede and Towns of Isakçe and Babadağ in the Northern Dobrudja", in Gilles Veinstein (éd.), Syncrétismes et hérésies dans l'Orient seldjoukide et ottoman (XIV -XVIII siècle), Paris, 2005, p. 291. 
[...] Les mécréants reprendront ces régions [...] C'est pour cela que tu dois être enterré ici [...] Il faut que ta tombe soit érigée dans ce pays, car les musulmans le reprendront $[\ldots]$ et sur tes cendres on érigera un mausolée. Ce miracle se produira parce que tu resteras ici : tout ce pays deviendra musulman $!^{14}$ »

De ce texte saisissant, il ressort que la présence des restes d'un saint musulman en terre infidèle est le gage d'une islamisation future de cette terre, cette inhumation comportant ainsi en elle-même, sous forme matérielle, l'annonce, la prophétie, d'un triomphe futur de l'islam. Ce triomphe se marquerait par l'érection d'un mausolée sur la tombe qui avait été préalablement cachée pour éviter les profanations des Infidèles.

Un épisode relatif aux premiers pas des Ottomans en Europe se conforme, de manière frappante, au schéma ainsi tracé. Il se rapporte aux derniers moments, en 1356, de Süleymân pacha, fils d'Orhân, conquérant de Gallipoli et chargé par son père d'étendre le beylik en Europe. Selon la chronique anonyme publiée par Giese, avant de mourir, Süleymân demande à ses compagnons de l'enterrer sur place (et non de le transporter à Bursa où se fixera la nécropole dynastique), mais de telle façon que les mécréants ne risquent pas de profaner sa tombe. Celle-ci doit donc rester dissimulée. De fait, peu après la mort de Süleymân, des mécréants arrivent par mer du côté de Qavaqtuzla et avant qu'une lutte acharnée ne s'engage entre mécréants et musulmans, les seconds cachent le cercueil sous un mur et accumulent des pierres, de manière à rendre la tombe invisible. Les chroniques rapporteront ensuite comment Murâd $1^{\mathrm{er}}$, après avoir pu passer pour la première fois les Dardanelles, soit dans l'hiver 1376-1377, en un temps où la Thrace était devenue musulmane, accomplit un pèlerinage sur la tombe de celui qui avait été le premier membre de la dynastie à mettre le pied sur la terre de Roumélie. Cette démarche impliquait qu'il ait, vingt ans après, « retrouvé » la tombe de son frère (on ne nous fournit pas de détails sur le déroulement de cette « réinvention »). Irène Beldiceanu-Steinherr concluait - reprenant les allégations de certains chroniqueurs - que, en dépit

14. Irène Mélikoff, La geste de Melik Dânişmend; Étude critique du Dânişmendnâme, II, Paris, 1960, p. 450. 
de l'attribution à Orhân dans la version conservée de l'acte de fondation, c'était Murâd $1^{\mathrm{er}}$ et non Orhân, qui, à la suite de ce pèlerinage, avait fait édifier le mausolée de Süleymân à Bolayır et institué le legs pieux destiné à son entretien ${ }^{15}$.

Le motif de la tombe musulmane cachée, prometteuse de conquête, que nous venons de rencontrer dans deux épisodes, au croisement, de l'histoire et de la légende, se retrouve encore dans un contexte, cette fois purement légendaire, lié au thème de la «Pomme d'or» ou «Pomme Rouge ${ }^{16}$. Dans des expressions populaires de ce thème, la conquête ultime des Turcs que symbolise la Pomme d'or a eu, comme il se doit, dans les époques antérieures, un précurseur dont la tombe, isolée en terre infidèle, attend, non plus l'arrivée d'un champion particulier (on n'est plus à l'époque où les sultans ottomans menaient leurs troupes en personne vers la victoire), mais, plus généralement et anonymement, celle des armées musulmanes qui viendront l'honorer comme il convient. Le plus ancien texte connu relevant de cette tradition est une lettre de 1662, présentée comme ayant été adressée à ses proches par un soldat des marches hongroises de l'empire, rentré de captivité chez les chrétiens ${ }^{17}$. L'auteur qui dit avoir été retenu prisonnier «à la Pomme d'or », y mentionne, entre autres éléments faisant partie de la description merveilleuse de la cité, au cœur de celle-ci, la présence du mausolée d'un certain Kâsım voyvoda. Ce dernier a pour origine un personnage historique: le chef de forces irrégulières ottomanes, chargé des raids en profondeur, lors de la campagne européenne de Soliman en 1532, connue sous le nom de «campagne d'Allemagne » (sefer- $i$ Alaman - une expédition marquée par des opérations en Hongrie, mais également des raids en Styrie lancés par le sultan qui avait campé devant Graz. Selon la chronique anonyme publiée par Friedrich Giese, pris au piège par des forces habsbourgeoises, il fut tué. Parmi les

15. Irène Beldiceanu-Steinherr, Recherches sur les actes des règnes des sultans Osman, Orkhan et Murad I, Munich, 1967, p. 135-140.

16. Mise au point et riche bibliographie chez Stéphane Yerasimos, «De l'arbre à la pomme : la généalogie d'un thème apocalyptique », in Benjamin Lellouch et Stéphane Yerasimos (eds.), op. cit., p. 153-192.

17. Cf. Orhan. Şaik Gökyay, «Kızıl Elma üzerine», Tarih ve Toplum, $\mathrm{n}^{\circ} 27,1986$, p. $9-10$. 
douze mille compagnons qui lui sont prêtés, certains connurent le même sort ; d'autres ne furent que blessés ${ }^{18}$. Personnage beaucoup plus modeste que les autres «précurseurs » que nous avons passés en revue, Kâsım voyvoda n'en est pas moins un martyr tombé en terre infidèle, et il devient le héros des irréguliers et des troupes des marches hongroises de l'empire. D'ailleurs, pour embellir sa statue, la tradition a préféré lier son martyre à un événement plus emblématique que la campagne de 1532, le siège de Vienne de 1529, comme on le constate, chez Evliyâ Çelebi notamment. D'ailleurs, sur ce même point de départ, l'auteur développe encore davantage le thème d'une présence musulmane au cœur des terres infidèles : il évoque la présence sur le versant des Alpes (Alaman dağı) d'une cité musulmane, peuplée par les descendants des compagnons rescapés de Kâsım voyvoda. Comme le note $\mathrm{S}$. Yerasimos, « le thème sert à entretenir l'espoir de retrouver les disparus, et, pose, en même temps, implicitement un jalon de la conquête » ${ }^{19}$.

Le même Kâsım réapparaît dans un autre texte, de même nature, mais de onze ans postérieur : une lettre envoyée par un prisonnier retenu dans la forteresse de Vienne, ville expressément assimilée à la Pomme-Rouge, et parvenue à la caserne de Chypre, le 6 novembre 1673 (soit quelque dix ans avant le second siège de Vienne) ${ }^{20}$. Kâsım voyvoda y est devenu Kâsım dede, c'est-à-dire qu'il a été doté d'un titre de derviche, plus particulièrement de derviche Bektachi. Lors du siège de Vienne par Soliman le Magnifique en 1529 , après que ce sultan eut eu en rêve une vision lui ordonnant de sacrifier 40000 martyrs, il se porte candidat en déclarant à son souverain :

«Commande ton serviteur Kâsım dede. Donne-lui quarante mille hommes ; nous tomberons en héros pour la religion évidente ». Kâsum et ses braves tombent en effet à la Pomme-Rouge après avoir affronté les mécréants. À côté de la forteresse de Vienne se trouve un couvent «avec une église à sept mille sept cents coupoles [soutenues par]

18. Friedrich Giese (éd.), Tevârih-i âl-i Osman, Breslau, 1922, p. 140-141.

19. Stéphane Yerasimos, art. cit., p. 185.

20. Pertev Naili Boratav, «La légende de la Pomme-Rouge et du Papede-Rome », in Le cuisinier et le philosophe. Hommage à Maxime Rodinson, Paris, 1982, p. 128. 
quatorze mille trois cents colonnes de marbre [...]. Le mausolée de Kâsım dede se trouve sous la coupole principale [...] C'est là que coule [le fleuve nommé] Danube. Cette coupole principale qu'on appelle La Pomme-Rouge a la forme d'une pomme en or; elle pèse quatre-vingt quatre kantar; c'est la raison pour laquelle on l'appelle la Pomme-Rouge. »

Par ailleurs, sur les quarante mille martyrs, quarante qui avaient demandé l'amân, avaient été laissés en vie. Les mécréants leur donnèrent quarante filles en mariage et ils sont à l'origine de la population d'une ville musulmane qui a grandi « au pied de la Pomme-Rouge ». Elle compte «soixante-dix grandes mosquées aux minarets de pierre et un millier de petites mosquées ». C'est une ville prospère et renommée, mais la population souffre néanmoins car les mécréants de la région lui imposent un lourd tribut. Cela fait conclure à l'auteur de la lettre : « Puisse Dieu le Très-Haut, par sa pitié pour le peuple de l'islam et par son affection pour ses Prophètes, les délivrer [de cette charge] ».

Ces lettres de captifs nous paraissent expliciter ce qui était resté implicite dans les instructions mortuaires relatives à Melik Danişmend ou à Süleymân Pacha : dans les premières, la tombe musulmane enfouie en territoire infidèle constituait une «promesse», une «prophétie » de la conquête islamique définitive du territoire. Dans les secondes, elle est une incitation à partir en campagne et à conquérir. L'obligation militaire est sans doute, de manière générale, un impératif religieux, mais l'impératif religieux est ici plus spécifique dans la mesure où il s'agit de mettre à l'abri une tombe musulmane des souillures des Infidèles, et également, par l'adjonction du thème des quarante rescapés faisant souche, de délivrer une ville musulmane des avanies et de l'oppression des Infidèles. Le lien entre tombe enfouie et idéologie de la conquête est particulièrement net dans le cas où l'on constate, comme nous venons de le voir, une identification entre la Pomme-Rouge, objectif ultime des armées ottomanes, et la coupole du mausolée de Kâsım dede à Vienne.

Dans la phase suivante du processus, celle de la conquête proprement dite, le sens de cette conquête est radicalement transformé : il ne s'agit plus d'un commencement absolu, d'une « ouverture » (ce qu'implique pourtant le terme arabe de feth employé pour exprimer 
la notion), et surtout pas d'une action purement offensive. Il s'agirait plutôt - plus ou moins nettement selon les cas - d'une reconquête et en tout cas d'une action fondamentalement défensive. À chaque fois, il y a eu un ou des précurseurs et il s'agit de délivrer leur tombe ainsi que, le cas échéant, d'affranchir leur progéniture.

Reprenons la liste des différents martyrs-précurseurs que nous avons rencontrés en cours de route.

Gazi Melik Danishmend est le précurseur des différents chefs turkmènes qui élimineront progressivement toute domination chrétienne de l'Anatolie pour intégrer ce pays au domaine de l'islam. Süleymân pacha est le précurseur de son frère Murâd qui se rend maitre de la Thrace et des autres membres de la dynastie qui s'empareront d'une grande partie de l'Europe. Abû Ayyub, porteétendard du Prophète, mort lors d'un des sièges arabes de Constantinople, dans les premiers temps de l'islam, est le précurseur de Mehmed II. Sarı Saltuk, chef spirituel des premiers Turcs venus d'Anatolie pour s'installer en Dobroudja, est le précurseur de Bâyezîd II dans ces régions du Bas-Danube. Il peut sembler plus paradoxal de présenter Ibn 'Arabî à la fois comme un martyr et comme un précurseur de Selîm $1^{\text {er }}$ en Syrie. Il faut cependant tenir compte d'une part des attaques dont il avait été l'objet de la part des oulémas damascènes, de son vivant et post mortem, si bien que sa tombe, quoiqu'en terre musulmane, avait été négligée et même insultée. Il faut se rappeler d'autre part que tout Espagnol qu'il fût par sa naissance à Murcie, le grand maitre soufi pouvait, dans une certaine mesure, être présenté comme anatolien et comme lié à la dynastie seljoukide dont les Ottomans se voulaient les successeurs : non seulement il avait en effet séjourné à Konya, mais c'était la ville du principal de ses disciples, Sadr al-Dîn Konevî qui était aussi son beau-fils (Ibn 'Arabî avait épousé sa mère) ; or Konevî, à en croire l'affirmation catégorique d'Evliyâ Çelebi, «était un descendant des Seldjoukides. ${ }^{21}$ »

21. «Konevî ise âl-i selçukiyân asrında zuhûr edüb Muhyiddîn'inü'l'Arabî'nin üveği evlâdıdır »; Evliyâ Çelebi Seyâhatnâmesi, Orhan Şaik Gökyay, I, p. 270. Le passage avait été omis dans l'édition ancienne d'Istanbul. 
Quant à Abû Hanîfa, grand docteur sunnite et fondateur d'une des quatre écoles juridiques orthodoxes, il pouvait être considéré comme précurseur des conquérants de Bagdad en 1534, Soliman le Magnifique et son très influent vizir, Ibrâhîm pacha (dont le rôle est soigneusement mis en valeur dans les récits de redécouverte de la tombe) dans la mesure où ces derniers, en conquérant l'ancienne capitale abbasside, la rendaient précisément à l'orthodoxie sunnite dont l'illustre juriste avait été un pilier.

On pouvait voir enfin dans la tante du Prophète tombée à Chypre une figure annonciatrice de la soumission définitive de l'île à l'islam à travers les défaites des garnisons vénitiennes devant les faits d'arme de Selîm II ou, plus exactement, de ses généraux.

\section{UN MODE DE LÉGITIMATION ET D'ISLAMISATION}

Interrogeons-nous pour finir sur la raison d'être d'un phénomène aussi récurrent : pourquoi faut-il que chaque conquête ou du moins de nombreuses conquêtes soient accompagnées de l' « invention » (au sens propre ou au sens figuré) de la tombe d'un précurseur de cette conquête ? On ne saurait écarter dans l'explication la simple force de l'imitation et le conformisme qui s'ensuit. Ne constate-t-on pas, à propos de nombreux rites monarchiques ottomans, qu'il suffit souvent qu'un souverain ait accompli, sous l'effet du hasard ou du moins d'un contexte particulier, tel acte dans telles circonstances, pour que celui-ci ne tarde pas à entrer dans l'«antique coutume ottomane » et à devenir pour ses successeurs un exemple à reproduire de toute nécessité ? Cette explication par l'exemple et l'effet de répétition n'est cependant, de toute évidence, pas suffisante. Elle ne va pas au fond des choses. À l'arrière-plan de ces manifestations diverses, on peut déceler en effet un seul et même schéma, une sorte d'archétype primitif dont il serait intéressant de rechercher des confirmations dans des modes archaïques d'appropriation du territoire, liés à l'organisation tribale et au nomadisme : un groupe n'est légitimement maitre d'un territoire donné que s'il s'agit du territoire de ses ancêtres et plus spécialement de son ancêtre mythique, 
le cas échéant éponyme. La présence de la tombe de cet ancêtre (incarné, comme nous l'avons vu, à travers des avatars variés) sur ce territoire apporte la preuve du bien-fondé de cette revendication. Telle est du moins du moins l'hypothèse à laquelle nous sommes conduit, face à ces comportements invariables et récurrents. Elle reste à approfondir.

D'autre part, le schéma primitif dont nous postulons l'existence est transcrit de toute façon dans des termes islamiques ou, plus exactement, dans les termes de l'islam turco-ottoman. Cette transposition islamique enrichit le schéma de nouvelles conséquences et de nouvelles significations. Si l'invention des tombes est, de toute évidence, une manière de légitimer la conquête, comment, plus précisément, la légitime-t-elle ?

Elle fait de la conquête un devoir de piété vis-à-vis d'un saint, voire d'un simple martyr musulman envers qui la solidarité s'impose à tout croyant. De manière plus générale et plus précise aussi, au regard du droit musulman, cette tombe, et à plus forte raison les mosquées et les minarets qui l'accompagnent (comme dans le cas de la légende de la ville de l'Alaman daght), sont autant de preuves que le pays où ils se trouvent a appartenu à un moment donné à l'islam. Or cette appartenance, fût-elle partielle, fût-elle éphémère, constituait en elle-même un fait irréversible. La conquête permettait ainsi de préserver les vestiges islamiques de la profanation et d'empêcher ce scandale : admettre que ce qui avait appartenu à l'islam ne lui appartienne plus. La présence d'antécédents faisait qu'on pouvait appliquer à toutes les conquêtes que nous avons évoquées l'argumentation que donnait Ebû Su'ûd efendi dans la fetvâ célèbre que nous avons citée plus haut, pour justifier non seulement la conquête ottomane de Chypre, mais la violation du 'ahdnâme accordé à Venise que représentait cette entreprise ${ }^{22}$.

22. Düzdağ, ibid. L'idée que Chypre avait été anciennement musulmane apparaît dans quelques écrits et propos des gouvernants ottomans du moment ; cf. la lettre de Selîm II à la population d'Andalousie in Abdeljelil Temimi, Le gouvernement ottoman et le problème morisque, Zaghouan, 1989, p. 19-22 ; Michel Lesure, «Notes et documents sur les relations vénéto-ottomanes, 1550-1573, II », Turcica, VIII/1, 1976, p. 134-135. 
Mais constater comme nous sommes en train de le faire que les Ottomans éprouvaient le besoin de justifier leur appropriation d'un territoire par des antécédents islamiques, c'est reconnaître aussi que d'autres raisons qu'on aurait pu croire a priori suffisantes, ne l'étaient pas en réalité. Ainsi le seul fait d'étendre, par l'adjonction d'un territoire neuf le dâr al-islâm au détriment du dâr al-harb, ne suffisait pas à légitimer une conquête. De même le pur droit de conquête, le droit du glaive, indépendamment de toute considération religieuse, dont on voit des gouvernants ottomans se faire les champions dans certaines circonstances (par exemple, en face d'émissaires habsbourgeois venus plaider à la Porte les droits de leur maitre sur la Hongrie ${ }^{23}$ ), n'était pas suffisant non plus. Or cette double constatation indique bien que les Ottomans ne se meuvent pas dans l'univers autiste d'un djihâd radical ou de la simple application du droit du glaive. Ils prennent en considération un monde plus complexe, où les droits respectifs des différents partenaires entrent en concurrence et où chacun doit défendre les siens au moyen d'un argumentaire plus sophistiqué. Dans cet argumentaire le principe d'antécédence joue un rôle fondamental. Pourtant, sa valeur, en l'occurrence, ne relève pas tant du droit du premier occupant (un principe universel valable aussi bien pour les Infidèles que pour les musulmans) qu'au caractère irréversible de toute emprise islamique (un principe qui ne vaut cette fois que pour les musulmans, mais dont ces derniers entendent imposer la reconnaissance à tous les autres).

Cette réalité générale est bien illustrée dans le cas d'un problème particulier qui surgit en 1542-1543 dans les steppes du nord de la mer Noire. Il s'agit en la circonstance, pour les émissaires du sultan Soliman, de tracer une frontière dans cette région entre les possessions de la Pologne - Lithuanie d'une part et des Ottomans et Tatars d'autre part. Soliman fait cette remarque significative au roi de Pologne, Sigismond Auguste, dans la lettre qu'il lui adresse à cette occasion :

«Dans les limites de la frontière en question [celle dont ses propres négociateurs préconisent le tracé] sont incluses les sépultures d'un grand nombre de personnes appartenant au peuple de l'islam ainsi

23. Joseph de Hammer, op. cit., V, p. 106-107, 192. 
qu'un grand nombre de vestiges de mosquées et de medrese. Ces éléments prouvent que cette frontière est la bonne. ${ }^{24}$ »

Moins expressément affirmé, le recours aux morts à des fins de revendication territoriale, reste cependant probable dans d'autres épisodes fameux.

Lors de sa campagne serbe de 1455, après la prise de Novo Brdo, à un moment où la région est définitivement rattachée aux territoires ottomans, Mehmed II va camper sur le site de la bataille de Kossovo, à l'endroit même où fut assassiné son ancêtre, Murâd $1^{\mathrm{er}}$, en 1389. Là, rapporte Nechrî, il fit des aumônes et des dons pour l'âme de son aïeul, tombé en martyr. Sur ce même site sera édifié un martyrium (meshhed), dont la date de construction nous est inconnue, mais dont l'existence est attestée, à partir du début du XVI ${ }^{\mathrm{e}}$ siècle ${ }^{25}$.

De la même façon un autre martyrium qui ne fut pas édifié sur le moment et dont la date exacte d'érection nous échappe encore (la plus ancienne mention repérée à ce jour est du 17 mars 1576), le türbe marquant l'emplacement du décès de Soliman le Magnifique en 1566, durant le siège de Szigetvàr, aura vraisemblablement correspondu au souci d'étayer la légitimité de la présence ottomane sur ce territoire hongrois qui restera toujours contestée ${ }^{26}$.

Ces tombes retrouvées, comme ces tombes (ou ces cénotaphes) érigés après coup qui, les uns comme les autres, ont pour rôle de perpétuer le souvenir d'un événement fondateur, apparaissent ainsi comme les instruments d'une légitimation dont le besoin se fait sentir dans un contexte donné. Mais il reste à se demander pourquoi

24. Cf. Gilles Veinstein, «L'occupation ottomane d'Očakov et le problème de la frontière lithuano-tatare », in Chantal Lemercier-Quelquejay, Senders E. Wimbush (eds.), Passé turco-tatar, Présent soviétique. Études offertes à Alexandre Bennigsen, Louvain-Paris, 1986, p. 123-155, en part., p. 147.

25. Neşri, Kitâb-i Cihân-nümâ, F. R. Unat et M. A. Köymen (eds.), II, Ankara, 1987, p. 720.

26. Cf. Nicolas Vatin, «Un türbe sans maître. Note sur la fondation et la destination du türbe de Soliman le Magnifique à Szigetvàr... », à paraître dans Turcica. Tous nos remerciements à l'auteur qui nous a communiqué son manuscrit encore inédit. 
ce mode de légitimation jouit d'une faveur si éclatante. La réponse est certainement à chercher dans la place tenue par le culte des tombeaux, sinon dans la doctrine musulmane (à toutes les époques, les docteurs un tant soit peu rigoristes ne cessent de dénoncer les pratiques et les croyances proprement magiques qui les fondent comme une déviation), du moins dans la vie religieuse effective des populations musulmanes, notamment turques.

Pour mesurer cette place, il suffit de lire les récits de voyageurs ottomans, qu'il s'agisse de Seydî 'Alî Re'îs ${ }^{27}$, d'Evliyâ Çelebi ou d'autres moins célèbres. On y constate que le passage à travers les différents villes et bourgs du dâr al-islâm s'accompagne immanquablement de visites aux tombeaux des saints, bienheureux et autres pieux personnages de toutes époques, qui peuvent s'y trouver (Soliman lui-même, avançant vers Bagdad, ne s'était-il pas arrêté en route, à Konya, sur la tombe de Mevlânâ ?). Bien souvent, ils n'ont rien de plus important ou rien d'autre à dire sur un lieu donné que de mentionner les morts illustres qui y sont enterrés. Il n'est, si l'on ose la formule, de tourisme que funéraire et donc que religieux.

Dès lors, les redécouvertes de tombes sont susceptibles d'un autre type d'interprétation (nullement exclusif du souci de légitimation) : comment mieux favoriser l'islamisation ou la ré-islamisation d'un territoire conquis qu'en donnant la possibilité aux musulmans qui s'y trouvent ou qu'on y établit, non seulement de le dominer (ce qu'implique la conquête) mais d'y trouver un aliment à leur foi ou un ferment d'orthodoxie? Sans doute doit-on y construire ou y rétablir des mosquées ou des medrese, mais cela ne suffit sans doute pas - sinon à l'accomplissement des devoirs religieux, du moins à l'exercice de la piété. Pour qu'un pays cesse d'appartenir au bilâd al-kufr et soit pleinement intégré au dâr al-islâm, il faut qu'il offre des saints à y vénérer et donc des tombeaux où puiser leur baraka. Telle pourrait être une autre fonction de ces tombeaux sacrés

27. Cf. Seyyidi 'Alî Re'îs, Le miroir des pays. Une anabase ottomane à travers l'Inde et l'Asie centrale, trad. et notes de Jean-Louis Bacqué-Grammont, Arles 1999. 
que les conquérants ottomans, et probablement d'autres conquérants musulmans de la même manière, prennent tant de soin à retrouver et à honorer sur les terres où ils entrent en vainqueurs.

gvs@ehess.fr

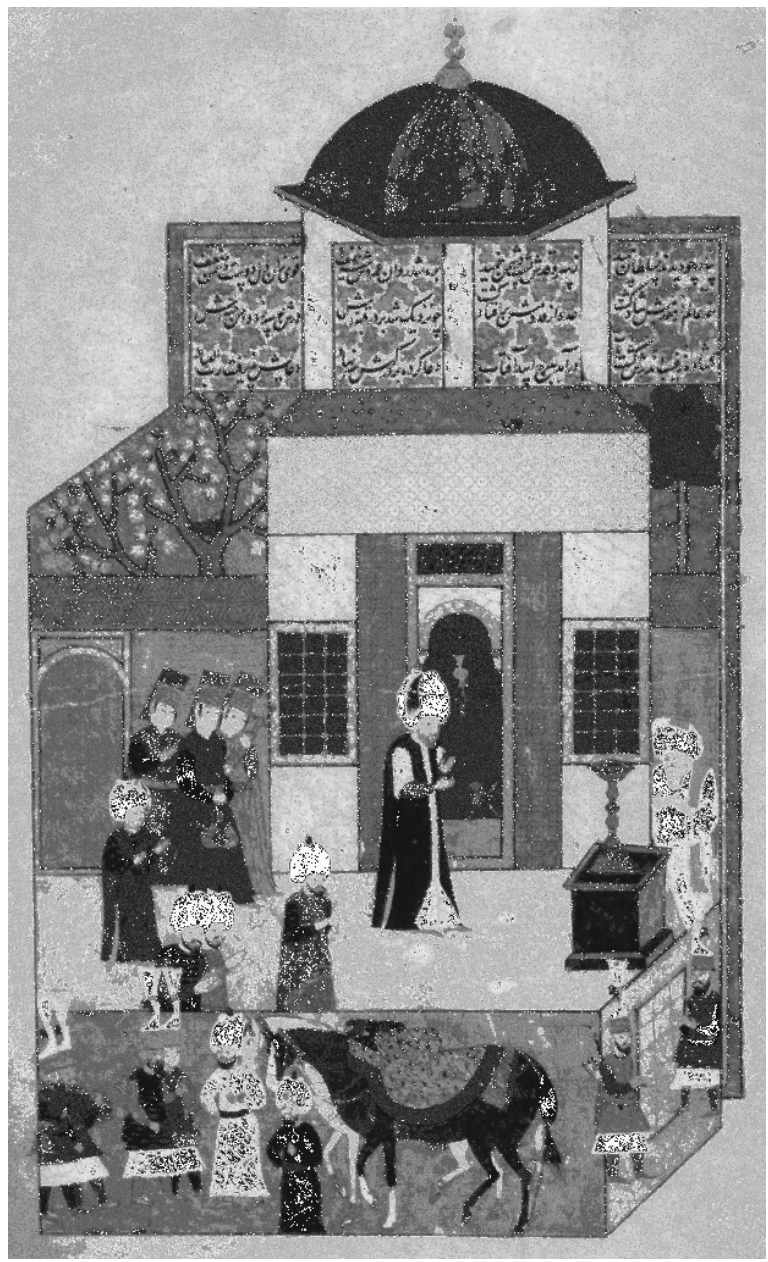

Visite de Soliman le Magnifique au tombeau d'Abû Ayyûb à côté d'Istanbul, extrait de Lokman, Tarih-i sutân Süleymân, Chester Beatty Library, Dublin, ms. 413, f. 38r. 